Journal of Sound and Vibration, Vol. 314, N ${ }^{\circ} 3-5,775-782$ (2008)

\title{
Harmonic balance approaches to the nonlinear oscillators in which the restoring force is inversely proportional to the dependent variable
}

A. Beléndez, D. Méndez, T. Beléndez, A. Hernández and M. L. Álvarez

Departamento de Física, Ingeniería de Sistemas y Teoría de la Señal.

Universidad de Alicante. Apartado 99. E-03080 Alicante. SPAIN

E-mail: a.belendez@ua.es

Corresponding author: A. Beléndez 
Phone: +34-96-5903651

Fax: +34-96-5903464 


\begin{abstract}
The second-order harmonic balance method is used to construct three approximate frequency-amplitude relations for a conservative nonlinear singular oscillator in which the restoring force is inversely proportional to the dependent variable. Two procedures are used to solve the nonlinear differential equation approximately. In the first the differential equation is rewritten in a form that does not contain the $y^{-1}$ expression, while in the second the differential equation is solved directly. The approximate frequency obtained using the second procedure is more accurate than the frequency obtained with the first one and the discrepancy between the approximate frequency and the exact one is lower than $1.28 \%$.
\end{abstract}

Keywords: Nonlinear oscillator; Harmonic balance method; Approximate frequency. 
Mickens [1] has recently analyzed the nonlinear differential equation

$$
\frac{d^{2} y}{d t^{2}}+\frac{1}{y}=0
$$

with initial conditions

$$
y(0)=A, \quad\left(\frac{d y}{d t}\right)_{t=0}=0
$$

Mickens has also shown that all the motions corresponding to Eq. (1) are periodic [1, 3]; the system will oscillate within symmetric bounds $[-A, A]$, and the angular frequency and corresponding periodic solution of the nonlinear oscillator are dependent on the amplitude $A$. Integration of Eq. (1) gives the first integral

$$
\frac{1}{2}\left(\frac{\mathrm{d} y}{\mathrm{~d} t}\right)^{2}+\log y=\log A
$$

where the integration constant was evaluated using the initial conditions of Eq. (2). From Eq. (3), the expression for the exact period, $T_{e x}(A)$, for the nonlinear oscillator given by Eq. (1) taking into account the initial conditions in Eq. (2) is

$$
T_{e x}(A)=4 \int_{0}^{A} \frac{d y}{\sqrt{2 \log (A / y)}}
$$

The transformation $y=A \mathrm{e}^{t^{2}}$ reduces this equation to the form

$$
T_{e x}(A)=4 \sqrt{2} A \int_{0}^{\infty} \mathrm{e}^{-t^{2}} d t=4 \sqrt{2} A \frac{\sqrt{\pi}}{2}=2 \sqrt{2 \pi} A
$$


From Eq. (5), the exact value for the angular frequency is given by the expression

$$
\omega_{e x}(A)=\frac{2 \pi}{T_{e x}(A)}=\frac{2 \pi}{2 \sqrt{2 \pi} A}=\frac{\sqrt{2 \pi}}{2 A}=\frac{1.2533141}{A}
$$

It is difficult to solve nonlinear differential equations and, in general, it is often more difficult to get an analytic approximation than a numerical one for a given nonlinear oscillatory system [3, 4]. There are many approaches for approximating solutions to nonlinear oscillatory systems. The most widely studied approximation methods are the perturbation methods [5]. The simplest and perhaps one of the most useful of these approximation methods is the Lindstedt-Poincare perturbation method, whereby the solution is analytically expanded in the power series of a small parameter [3]. To overcome this limitation, many new perturbative techniques have been developed. Modified Lindstedt-Poincaré techniques [6-8], homotopy perturbation method [9-15] or linear delta expansion [16-18] are only some examples of them. A recent detailed review of asymptotic methods for strongly nonlinear oscillators can be found in reference [4]. The harmonic balance method is another procedure for determining analytical approximations to the periodic solutions of differential equations by using a truncated Fourier series representation [3, 19-25]. This method can be applied to nonlinear oscillatory systems where the nonlinear terms are not small and no perturbation parameter is required.

The main objective of this paper is to approximately solve Eq. (1) by applying the harmonic balance method, and to compare the approximate frequency obtained with the exact one and with another approximate frequency obtained applying the harmonic balance method to the same oscillatory system but rewriting Eq. (1) in a way suggested previously by Mickens [1]. The approximate frequency derived here is more accurate and closer to the exact solution. The error in the resulting frequency is reduced and the maximum relative error is less than $1.3 \%$ for all values of $A$.

In order to approximately solve Eq. (1), Mickens has rewritten this equation in a form that does not contain the $y^{-1}$ expression [1] 


$$
y \frac{d^{2} y}{d t^{2}}+1=0, \quad y(0)=A, \quad\left(\frac{d y}{d t}\right)_{t=0}=0
$$

It is possible to solve Eq. (7) by applying the harmonic balance method. Following the lowest order harmonic balance method, a reasonable and simple initial approximation satisfying the conditions in Eq. (7) would be

$$
y_{1}(t)=A \cos \omega t
$$

The substitution of Eq. (8) into Eq. (7) gives

$$
-A^{2} \omega^{2} \cos ^{2} \omega t+1=0
$$

then expanding and simplifying the resulting expression gives

$$
-\frac{1}{2} A^{2} \omega^{2}+1+(\text { higher }- \text { order harmonics })=0
$$

and the solution for the angular frequency, $\omega_{M 1}(A)$, is

$$
\omega_{M 1}(A)=\frac{\sqrt{2}}{A}=\frac{1.414214}{A}
$$

and the percentage error of this approximate frequency in relation to the exact one is

$$
\text { percentage error }=\left|\frac{\omega_{e x}-\omega_{M 1}}{\omega_{e x}}\right| \cdot 100=12.8 \%
$$

Mickens [1] also used the second-order harmonic balance approximation 


$$
y_{2}(t)=A_{2} \cos \omega t+B_{2} \cos 3 \omega t
$$

to the periodic solution of Eq. (7). Substitution of Eq. (13) into Eq. (7), simplifying the resulting expression and equating the coefficients of the lowest harmonics to zero gives two equations and taking into account that $A=A_{2}+B_{2}$, Mickens [1] obtained $A_{2}=10 A / 9$ and $B_{2}=-A / 9$, and the second-order approximate solution (Eq. (13)) to Eq. (7) can be written as follows

$$
y_{M 2}(t)=\frac{10}{9} A \cos \left(\omega_{M 2} t\right)-\frac{1}{9} A \cos \left(3 \omega_{M 2} t\right)
$$

where the second-order approximate frequency, $\omega_{M 2}(A)$, is given by

$$
\omega_{M 2}(A)=\frac{\sqrt{162}}{10 A}=\frac{1.272792}{A}
$$

and the percentage error is

$$
\text { percentage error }=\left|\frac{\omega_{e x}-\omega_{M 2}}{\omega_{e x}}\right| \cdot 100=1.55 \%
$$

As we pointed out previously, the main objective of this paper is to solve Eq. (1) instead of Eq. (7) by applying the harmonic balance method. Substitution of Eq. (8) into Eq. (1) gives

$$
-A \omega^{2} \cos \omega t+\frac{1}{A \cos \omega t}=0
$$

In order to apply the first order harmonic balance method to Eq. (17) we have to expand Eq. (17) and to set the coefficient of $\cos \omega t$ (the lowest order harmonic) equal to zero. To take this, firstly we do the following Fourier series expansion 


$$
\frac{1}{A \cos \omega t}=\sum_{n=0}^{\infty} a_{2 n+1} \cos [(2 n+1) \omega t]=a_{1} \cos \omega t+a_{3} \cos 3 \omega t+\ldots
$$

where the first term of this expansion can be obtained by means of the following equation

$$
a_{1}=\frac{4}{\pi} \int_{0}^{\pi / 2} \frac{1}{A \cos \theta} \cos \theta \mathrm{d} \theta=\frac{2}{A}
$$

Substituting Eq. (18) into Eq. (17) and taking into account Eq. (19) gives

$$
\left\lfloor-\omega^{2}+\frac{2}{A}\right\rfloor \cos \omega t+(\text { higher }- \text { order harmonics })=0
$$

For the lowest order harmonic to be equal to zero, it is necessary to set the coefficient of $\cos \omega t$ equal to zero in Eq. (20), then

$$
\omega_{1}(A)=\frac{\sqrt{2}}{A}=\frac{1.4142}{A}
$$

Consequently, in this limit, the low-order harmonic balance method applied to Eq. (1) gives exactly the same results as the low-order harmonic balance method applied to Eq. (7).

In order to obtain the next level of harmonic balance, we express the periodic solution to Eq. (1) with the assigned conditions in Eq. (2) in the form of [19-22]

$$
y_{2}(t)=y_{1}(t)+u(t)
$$

where $u(t)$ is the correction part which is a periodic function of $t$ of period $2 \pi / \omega$ and 


$$
u(0)=0, \quad\left(\frac{d u}{d t}\right)_{t=0}=0
$$

Substituting Eq. (22) into Eq. (1) gives

$$
\frac{\mathrm{d}^{2} y_{1}}{\mathrm{~d} t^{2}}+\frac{\mathrm{d}^{2} u}{\mathrm{~d} t^{2}}+\frac{1}{y_{1}(t)+u(t)}=0
$$

$\mathrm{Wu}$ and Lim [19-21] presented an approach by combining the harmonic balance method and linearization of nonlinear oscillation equation with respect to displacement increment only, $u(t)$. This harmonic balance approach will be used to approximately solve Eq. (24). Linearizing the governing Eq. (24) with respect to the correction $u(t)$ at $y_{1}(t)$ leads to

$$
\frac{\mathrm{d}^{2} y_{1}}{\mathrm{~d} t^{2}}+\frac{\mathrm{d}^{2} u}{\mathrm{~d} t^{2}}+\frac{1}{y_{1}(t)}-\frac{u(t)}{y_{1}^{2}(t)}=0
$$

and

$$
u(0)=0, \quad\left(\frac{d u}{d t}\right)_{t=0}=0
$$

To obtain the second approximation to the solution, $u(t)$ in Eq. (22), which must satisfy the initial conditions in Eq. (26), we take into account the second-order harmonic balance approximation in Eq. (13) which can be written as follows

$$
y_{2}(t)=A_{2} \cos \omega t+B_{2} \cos 3 \omega t=A \cos \omega t-B_{2} \cos \omega t+B_{2} \cos 3 \omega t=A \cos \omega t+B_{2}(\cos 3 \omega t-\cos \omega t)
$$

where we have taken into account that $A=A_{2}+B_{2}$. From Eqs. (8), (22) and (27) we can see that $u(t)$ takes the form 


$$
u(t)=B_{2}(\cos 3 \omega t-\cos \omega t)
$$

where $B_{2}$ is a constant to be determined.

Substituting Eqs. (22), (8) and (28) into Eq. (25), expanding the expression in a trigonometric series and setting the coefficients of the terms $\cos \omega t$ and $\cos 3 \omega t$ equal to zero, respectively, leads to

$$
-\left(A-B_{2}\right) \omega^{2}+\frac{2}{A^{2}}\left(A+2 B_{2}\right)=0
$$

and

$$
\frac{-2 A-8 B_{2}-9 B_{2} A^{2} \omega^{2}}{A^{2}}=0
$$

From Eqs. (29) and (30) we can obtain $B_{2}$ and $\omega_{2}$ as follows

$$
\begin{gathered}
B_{W L 2}=\frac{A}{14}(\sqrt{22}-6)=-0.093542 A \\
\omega_{W L 2}(A)=\sqrt{\frac{2 A+8 B_{W L 2}}{-9 B_{W L 2} A^{2}}}=\frac{1.2193273}{A}
\end{gathered}
$$

With this value for $B_{2}$, Eq. (27) can be written

$$
y_{W L 2}(t)=\frac{20-\sqrt{22}}{14} A \cos \left(\omega_{W L 2} t\right)+\frac{\sqrt{22}-6}{14} A \cos \left(3 \omega_{W L 2} t\right)
$$

The percentage error for the second order approximate frequency is 


$$
\text { percentage error }=\left|\frac{\omega_{e x}-\omega_{W L 2}}{\omega_{e x}}\right| \cdot 100=2.71 \%
$$

which is higher than the percentage error for the second approximate frequency obtained by Mickens when harmonic balance method is applied to Eq. (7).

As we can see substitution of Eq. (22) into Eq. (1) does not give the same result as substitution of Eq. (8) into Eq. (7) and application of the second-order harmonic balance method to Eq. (7) give a more accurate frequency than application of Wu and Lim's approach to Eq. (1). These questions have been analyzed in detail in references [23] and [25] for other oscillators analyzed by first-order harmonic balance method and one would wait to obtain better results when the harmonic balance method is applied to Eq. (1) instead of to Eq. (7). This would be due to the fact that when we substitute Eq. (13) into Eq. (7) we obtain an equation that includes only three even powers of $\cos \omega t: 1\left(\cos ^{0} \omega t\right)$, $\cos ^{2} \omega t$ and $\cos ^{4} \omega t$ and then there are only three contributions to the coefficient of the first term $1(\cos 0 \omega t)$, from $1\left(\cos ^{0} \omega t\right), \cos ^{2} \omega t$ and $\cos ^{4} \omega t$, and two contributions to the coefficient of the second harmonic $\cos (2 \omega t)$, from $\cos ^{2} \omega t$ and $\cos ^{4} \omega t$, Therefore, substituting Eq. (13) into Eq. (7) produces only three terms, 1, $\cos (2 \omega t)$ and $\cos (4 \omega t)$. However, Eq. (17) includes all odd powers of $\cos \omega t$, which $\operatorname{are} \cos ^{2 n+1} \omega t$ with $n=0,1,2$, $\ldots, \infty$, and then there are infinite contributions to the coefficient of the first harmonic $\cos \omega t$, that is, 1 from $\cos \omega t, 3 / 4$ from $\cos ^{3} \omega t, 5 / 8$ from $\cos ^{5} \omega t, \ldots, 2^{-2 n}\left(\begin{array}{c}2 n+1 \\ n\end{array}\right)$ from $\cos ^{2 n+1} \omega t$, and so on. Therefore, substituting Eq. (27) into Eq. (1) produces the infinite set of higher harmonics, $\cos \omega t, \cos 3 \omega t, \ldots, \cos [(2 n+1) \omega t]$, and so on, and the second-order angular frequency in Eq. (32) would have to be more accurate than the frequency given in Eq. (15). But we obtained the opposite result. The reason is that we have not applied the exact second-harmonic balance method to Eq. (1), but a linearized approximation to this method.

In order to verify this affirmation, we consider a new approach to obtain higherorder approximations using harmonic balance method. Instead of considering the assumption in Eq. (25), first we do the following series expansion 


$$
\frac{1}{y_{1}(t)+u(t)}=\frac{1}{y_{1}(t)\left[1+y_{1}^{-1}(t) u(t)\right]}=\sum_{n=0}^{\infty}(-1)^{n} \frac{u^{n}(t)}{y_{1}^{n+1}(t)}
$$

and substituting Eq. (35) into equation (1) gives

$$
\frac{\mathrm{d}^{2} y_{1}}{\mathrm{~d} t^{2}}+\frac{\mathrm{d}^{2} u}{\mathrm{~d} t^{2}}+\sum_{n=0}^{\infty} \frac{(-1)^{n} u^{n}(t)}{y_{1}^{n+1}(t)}=0
$$

To obtain the second approximation to the solution, $u(t)$ in Eq. (22), which must satisfy the initial conditions in Eq. (23), takes the form

$$
u(t)=B_{2}(\cos 3 \omega t-\cos \omega t)=4 B_{2}\left(\cos ^{3} \omega t-\cos \omega t\right)
$$

where $c_{2}$ is a constant to be determined.

Substituting Eqs. (8), (22) and (37) into Eq. (36) gives

$$
-\omega^{2}\left(A-B_{2}\right) \cos \omega t-9 \omega^{2} B_{2} \cos 3 \omega t+\sum_{n=0}^{\infty} \frac{4^{n} B_{2}^{n}\left(1-\cos ^{2} \omega t\right)^{n}}{A^{n+1} \cos \omega t}=0
$$

The formula that allows us to obtain $\left(1-\cos ^{2} \omega t\right)^{n}$ is

$$
\left(1-\cos ^{2} \omega t\right)^{n}=\sum_{k=0}^{n}\left(\begin{array}{l}
n \\
k
\end{array}\right)\left(-\cos ^{2} \omega t\right)^{k}=\sum_{k=0}^{n} \frac{(-1)^{k} n !}{k !(n-k) !} \cos ^{2 k} \omega t
$$

Substituting Eq. (39) into Eq. (38) gives 
$-\omega^{2}\left(A-B_{2}\right) \cos \omega t+9 \omega^{2} B_{2} \cos 3 \omega t+\sum_{n=0}^{\infty} \frac{4^{n} n ! B_{2}^{n}}{A^{n+1}} \sum_{k=0}^{n} \frac{(-1)^{k}}{k !(n-k) !} \cos ^{2 k-1} \omega t=0$

It is possible to state the following Fourier series expansion

$$
\cos ^{2 k-1} \omega t=\sum_{j=0}^{\infty} b_{2 j+1}^{(k)} \cos [(2 j+1) \omega t]=b_{1}^{(k)} \cos \omega t+b_{3}^{(k)} \cos 3 \omega t+\ldots
$$

where

$$
b_{2 j+1}^{(k)}=\frac{4}{\pi} \int_{0}^{\pi / 2} \cos ^{2 k-1} \theta \cos [(2 j+1) \theta] \mathrm{d} \theta=\frac{2(k-1) ! \Gamma\left(k+\frac{1}{2}\right)}{\sqrt{\pi}(k-j-1) !(j+k) !}
$$

and where $\Gamma(z)$ is the Euler gamma function [26].

Substituting Eqs. (41) and (42) into Eq. (40), we obtain

$-\omega^{2}\left(A-B_{2}\right) \cos \omega t-9 \omega^{2} B_{2} \cos 3 \omega t+$

$$
+\sum_{n=0}^{\infty} \frac{4^{n} n ! B_{2}^{n}}{A^{n+1}} \sum_{k=0}^{n} \frac{(-1)^{k}}{k !(n-k) !} \sum_{j=0}^{k} \frac{2(k-1) ! \Gamma\left(k+\frac{1}{2}\right)}{\sqrt{\pi}(k-j-1) !(j+k) !} \cos [(2 j+1) \omega t]=0
$$

and setting the coefficients of the resulting items $\cos \omega t(j=0)$ and $\cos 3 \omega t(j=1)$ equal to zero, respectively, yields

$$
\begin{aligned}
& -\omega^{2}\left(A-B_{2}\right)+\sum_{n=0}^{\infty} \frac{4^{n} n ! B_{2}^{n}}{A^{n+1}} \sum_{k=0}^{n} \frac{(-1)^{k} 2 \Gamma\left(k+\frac{1}{2}\right)}{\sqrt{\pi}(k !)^{2}(n-k) !}=0 \\
& -9 \omega^{2} B_{2}+\sum_{n=0}^{\infty} \frac{4^{n} n ! B_{2}^{n}}{A^{n+1}} \sum_{k=0}^{n} \frac{(-1)^{k} 2(k-1) \Gamma\left(k+\frac{1}{2}\right)}{\sqrt{\pi} k !(k+1) !(n-k) !}=0
\end{aligned}
$$


which can be written as follows

$$
\begin{gathered}
-\omega^{2}\left(A-B_{2}\right)+\frac{2}{A} \sqrt{\frac{A}{A-4 B_{2}}}=0 \\
-9 \omega^{2} B_{2}+\frac{2 A+2 B_{2}-2 \sqrt{A^{2}-4 A B_{2}}}{B_{2} \sqrt{A^{2}-4 A B_{2}}}=0
\end{gathered}
$$

In Eq. (47) the following relations have to be taken into account

$$
\begin{gathered}
\sum_{k=0}^{n} \frac{(-1)^{k} 2 \Gamma\left(k+\frac{1}{2}\right)}{\sqrt{\pi}(k !)^{2}(n-k) !}=\frac{2 \Gamma\left(k+\frac{1}{2}\right)}{\sqrt{\pi}(n !)^{2}} \\
\sum_{n=0}^{\infty} \frac{4^{n} n ! B_{2}^{n}}{A^{n+1}} \frac{2 \Gamma\left(k+\frac{1}{2}\right)}{\sqrt{\pi}(n !)^{2}}=\frac{2}{A} \sqrt{\frac{A}{A-4 B_{2}}}
\end{gathered}
$$

while in Eq. (48) the following expressions have been considered

$$
\begin{aligned}
& \sum_{k=0}^{n} \frac{(-1)^{k} 2(k-1) \Gamma\left(k+\frac{1}{2}\right)}{\sqrt{\pi} k !(n-k) !(1+k) !}=-\frac{2\left[\Gamma\left(n+\frac{1}{2}\right) n !+2(n-1) ! \Gamma\left(n+\frac{3}{2}\right)\right]}{\sqrt{\pi}(n+1) ! n !(n-1) !} \\
& -\sum_{n=0}^{\infty} \frac{(-1)^{n} 4^{n} n ! c_{2}^{n}}{A^{n+1}} \frac{2\left[\Gamma\left(n+\frac{1}{2}\right) n !+2(n-1) ! \Gamma\left(n+\frac{3}{2}\right)\right]}{\sqrt{\pi}(n+1) ! n !(n-1) !}=\frac{-2 A+2 B_{2}+2 \sqrt{A^{2}-4 A B_{2}}}{B_{2} \sqrt{A^{2}-4 A B_{2}}}
\end{aligned}
$$

These results have been obtained using Mathematica ${ }^{\circledR}$.

From Eqs. (46) and (47) and once again by using Mathematica ${ }^{\circledR}$ we can obtain $\omega_{2}$ 
and $B_{2}$ as follows

$$
\begin{gathered}
B_{2}=-\frac{A}{50}\left[(2159+225 \sqrt{106})^{1 / 3}-6-\frac{89}{(2159+225 \sqrt{106})^{1 / 3}}\right\rfloor=0.10158074 A \\
\omega_{2}(A)=\sqrt{\frac{2}{A\left(A-B_{2}\right)} \sqrt{\frac{A}{A-4 A B_{2}}}}=\frac{1.237330058}{A}
\end{gathered}
$$

With this value for $B_{2}$, Eq. (22) can be written

$$
y_{2}(t)=1.101581 A \cos \left(\omega_{2} t\right)-0.101581 A \cos \left(3 \omega_{2} t\right)
$$

The percentage error for the second order approximate frequency is

$$
\text { percentage error }=\left|\frac{\omega_{e x}-\omega_{2}}{\omega_{e x}}\right| \cdot 100=1.275 \%
$$

As we can see, this error is lower than the error obtained by Mickens (1.55\%) and we can conclude this is the percentage error obtained when the second-order harmonic balance method is exactly applied to Eq. (1).

The second order harmonic balance method was used to obtain three approximate frequencies for a nonlinear singular oscillator. The first approximate frequency, $\omega_{M 2}$, was obtained by rewriting the nonlinear differential equation in a form that does not contain the $y^{-1}$ term; while the second and the third ones, $\omega_{W L 2}$ and $\omega_{2}$, were obtained by solving the nonlinear differential equation containing the $y^{-1}$ term. The second-order approximate frequency $\omega_{W L 2}$ is obtained by using the approach by Wu and Lim [19-21]. This approach can be described as a linearisation of the harmonic balance method, and works pretty well for the chosen problems. Because the harmonic balance method does not eliminate the 
secular terms systematically, it is difficult to obtain second and higher order approximate solutions by the harmonic balance method. But this approach eliminates this difficulty and may be applied to other nonlinear oscillators. We can conclude that equations (52) and (53) are valid for the complete range of oscillation amplitude, including the limiting cases of amplitude approaching zero and infinity. Excellent agreement of the approximate frequencies with the exact value was demonstrated, and discussed, and the discrepancy between the third approximate frequency, $\omega_{2}$, and the exact value never exceeds $1.28 \%$. The approximate frequency, $\omega_{2}$, derived here is the best frequency that can be obtained using the first-order harmonic balance method, and the maximum relative error was reduced as compared with approximate frequencies $\omega_{M 2}$ and $\omega_{W L 2}$. Finally, we discussed the reason why the accuracy of the approximate frequency, $\omega_{2}$, is better than that of the frequency $\omega_{M 2}$ obtained by Mickens. This reason is related to the number of harmonics that application of the second-order harmonic balance method produces for each differential equation solved.

\section{Acknowledgements}

This work was supported by the "Ministerio de Educación y Ciencia", Spain, under project FIS2005-05881-C02-02, and by the "Generalitat Valenciana", Spain, under project ACOMP/2007/020. 


\section{References}

[1] R. E. Mickens, "Harmonic balance and iteration calculations of periodic solutions to $\ddot{y}+y^{-1}=0 ”$, J. Sound Vib. 306, 968-972 (2007).

[2] J. R. Acton and P. T. Squire, Solving Equations with Physical Inderstanding, Adam Hilger, Bristol and Boston, 1985 (Chapter 5).

[3] R. E. Mickens, Oscillations in Planar Dynamics Systems (World Scientific, Singapore 1996).

[4] J. H. He, "Some asymptotic methods for strongly nonlinear equations", Int. J. Modern Phys. B 10, 1141-99 (2006).

[5] A. H. Nayfeh, Problems in Perturbations (Wiley, New York 1985).

[6] J. H. He, "Modified Lindstedt-Poincare methods for some non-linear oscillations. Part I: expansion of a constant”, Int. J. Non-linear Mech. 37, 309-314 (2002).

[7] T. Özis and A. Yildirim, "Determination of periodic solution for a $u^{1 / 3}$ force by He's modified Lindstedt-Poincaré method", J. Sound Vib. 301, 415-419 (2007).

[8] D. H. Shou and J. H. He, "Application of parameter-expanding method to strongly nonlinear oscillators", Int. J. Non-linear Sci. Numer. Simulation 8 (1), 121-124 (2007).

[9] J. H. He, "Homotopy perturbation method for bifurcation on nonlinear problems", Int. J. Non-linear Sci. Numer. Simulation. 6, 207-208 (2005).

[10] F. Shakeri and M. Dehghan, "Inverse problem of diffusion by He's homotopy perturbation method", Phys. Scr. 75, 551-556 (2007). 
[11] D. D. Ganji and A. Sadighi, “Application of He's homotopy-perturbation method to nonlinear coupled systems of reaction-diffusion equations", Int. J. Non-linear Sci. Numer. Simulation 7 (4), 411-418 (2006).

[12] A. Beléndez, A. Hernández, T. Beléndez and A. Márquez, "Application of the homotopy perturbation method to the nonlinear pendulum", Eur. J. Phys. 28, 93-104 (2007).

[13] A. Beléndez, A. Hernández, T. Beléndez, E. Fernández, M. L. Álvarez and C. Neipp, "Application of He's homotopy perturbation method to the Duffing-harmonic oscillator", Int. J. Non-linear Sci. Numer. Simulation 8 (1), 79-88 (2007).

[14] M. S. H. Chowdhury and I. Hashim, "Solutions of a class of singular second-order IVPs by homotopy-perturbation method", Phys. Lett. A 365, 439-447 (2007).

[15] T. Özis and A. Yildirim, “A comparative Study of He's homotopy perturbation method for determining frequency-amplitude relation of a nonlinear oscillator with discontinuities", Int. J. Non-linear Sci. Numer. Simulation 8 (2), 243-248 (2007).

[16] P. Amore and A. Aranda, "Improved Lindstedt-Poincaré method for the solution of nonlinear problems", J. Sound. Vib. 283, 1115-1136 (2005).

[17] P. Amore and F. M. Fernández, "Exact and approximate expressions for the period of anharmonic oscillators", Eur. J. Phys. 26, 589-601 (2005).

[18] P. Amore, A. Raya and F. M. Fernández, "Alternative perturbation approaches in classical mechanics", Eur. J. Phys. 26, 1057-1063 (2005).

[19] B. S. Wu and C. W. Lim, "Large amplitude nonlinear oscillations of a general conservative system", Int. J. Non-linear Mech. 39, 859-870 (2004).

[20] C. W. Lim and B. S. Wu, "Accurate higher-order approximations to frequencies of 
nonlinear oscillators with fractional powers", J. Sound Vib. 281, 1157-1162 (2005).

[21] C. W. Lim and B. S. Wu, "A new analytical approach to the Duffing-harmonic oscillator", Phys. Lett. A 311, 365-373 (2003).

[22] A. Beléndez, A. Hernández, A. Márquez, T. Beléndez and C. Neipp, “Analytical approximations for the period of a simple pendulum”, Eur. J. Phys. 27, 539-551 (2006).

[23] H. Hu and J. H. Tang, "Solution of a Duffing-harmonic oscillator by the method of harmonic balance", J. Sound Vib. 294, 637-639 (2006).

[24] A. Beléndez and C. Pascual, "Harmonic balance approach to the periodic solutions of the (an)harmonic relativistic oscillator", Phys. Lett. A 371, 371, 291-299 (2007).

[25] A. Beléndez, A. Hernández, T. Beléndez, M.L. Álvarez, S. Gallego, M. Ortuño and C. Neipp "Application of the harmonic balance method to a nonlinear oscillator typified by a mass attached to a stretched wire", J. Sound Vib. 302, 1018-1029 (2007).

[26] M. Abramowitz and I. A. Stegun, (Eds.), "Gamma (Factorial) Function" and "Incomplete Gamma Function" $\$ 6.1$ and 6.5 in Handbook of Mathematical functions with Formulas, Graphs and Mathematical Tables. New York: Dover, pp. 258 and 263, 1972. 